\title{
Dose-Mutation Relationships in Ultraviolet-Induced Reversion from Auxotrophy in Escherichia coli
}

\author{
By RUTH F. HILL \\ Radiological Research Laboratory, Department of Radiology, Columbia University, \\ Nere York, U.S.A.
}

(Received 1 June 1962)

SUMMARY

Curves for the absolute yield of revertants as a function of ultraviolet (uv) radiation dose were obtained after post-irradiation incubation in saline and after exposure to reactivating light. The curves exhibited plateau regions and final slopes which were equal to the slopes of the survival curves for the auxotrophic Escherichia coli parent. These features may be due to a saturation of the mutagenic effect at low doses. This saturation would be the result of $(a)$ heterogeneity in the unirradiated population with respect to the amount of protein and RNA synthesis which can occur before DNA replicates in the irradiated organisms, combined with (b) limitation of the observable yield by the selection method. The observable yield would be derived from the fraction of the population most resistant to the lethal effect of radiation.

\section{INTRODUCTION}

The mechanism by which ultraviolet (u.v.) radiation produces mutations to prototrophy in amino acid-requiring strains of bacteria has been the subject of many recent investigations (Witkin, 1958, 1961; Lieb, 1960; Weatherwax \& Landman, 1960; Doudney \& Haas, 1958, 1960a, $b$; Haas \& Doudney, 1959; Doudney, 1961). Briefly, u.v.-radiation produces a large number of potential prototrophs. The fraction which is realized (the yield) is determined by the amount of protein and RNA metabolism which takes place in the irradiated auxotrophic organisms during the period before DNA synthesis. During this critical period, the yield can be decreased by treatments which inhibit protein or RNA synthesis. Treatments which prolong this critical period increase the yield.

Two hypotheses have been advanced to explain these findings. The first suggests that the initial effect of u.v.-radiation is some unknown kind of damage to DNA and that in the absence of adequate protein and RNA metabolism, the damage is repaired. Failure of repair results in the stabilization of the damage in the form of a mutation when DNA replicates (Witkin, 1961). The second hypothesis is that the initial damage is not to DNA but to a precursor of RNA. When this modified precursor becomes part of RNA, it causes a mistake in the structure of DNA (Doudney, 1961).

The following report concerns the relationship between the u.v. dose and the yield of mutations. The effects on this relationship of various post-irradiation treatments and of the method of scoring mutants will be described. 


\section{METHODS}

Bacterial strains. The experiments were performed with tryptophan-requiring strains of Escherichia coli strain B/r, mostly with a strain which will be referred to as WP2. This strain had been passed from Dr E. M. Witkin to Professor F. J. Ryan, to Dr S. B. Greer before we obtained it. A few experiments were done with a strain obtained directly from Dr Witkin and therefore designated as WWP2. Both strains are apparently blocked at the same point, namely, a step before anthranilic acid in the synthesis of tryptophan. Neither strain can utilize shikimic acid. The growth of these strains in anthranilic acid is restricted. The maximum rate is obtained with 0.5-1 $\mu \mathrm{g} . / \mathrm{ml}$. and the rate decreases with concentrations greater than this. Inhibition of the growth of an auxotroph by an excess of the same metabolite which is required was pointed out by Soboren \& Nyc (1961). Although WP2 and WWP2 seem to be identical in their growth behaviour, their response to u.v. irradiation is different. The yield for WWP2 is 4-5 times that for WP2.

Irradiation. The techniques for growing cultures, irradiating and determining the survival of the parent and the yield of revertants were those described by Witkin (1958). Unless stated otherwise, stationary-phase cultures were used. Unfractionated doses were always given. Plating was done on SEM (minimal medium $+5 \%$ nutrient broth, solidified with unwashed agar) and/or, $\mathbf{M} \mathbf{M}$ (minimal medium $\mathbf{E}$ solidified with washed agar; Vogel \& Bonner, 1956).

The apparatus used for u.v.-irradiation and for photoreversal was described by Hill \& Simson (1961). Post-irradiation incubation was carried out by simply transferring the saline suspension from the Petri dish in which it had been irradiated to a test tube and immersing the test tube in the $37^{\circ}$ water-bath of the photoreversal apparatus for $1 \mathrm{hr}$. In experiments in which the effect of incubation, with and without exposure to the light, was to be determined, the irradiated culture was divided into two test tubes. One of these was wrapped in aluminium foil before immersion in the water-bath. Although the effect of the reversing light was actually the combined effect of the light and the incubation, no correction was made. Since it was not feasible to obtain entire dose/mutation curves with and without posttreatments in a single experiment, limited sections of dose range were used and the results normalized to the data for overlapping doses used in different experiments.

\section{RESULTS}

Figure 1 shows the effects of incubation in saline and of exposure to light as functions of u.v. dose when the yield was measured on SEM. With both types of post-treatment, the absolute yield of revertants was decreased in the low dose range, in agreement with other reports (Doudney \& Haas, $1960 a$; Lieb, 1960). In the very high dose range, the revertant yield seemed to decline at the same rate as the auxotrophs were killed. To determine whether this was true, repeated experiments were done in this range. No difference in rate was detected. It may be concluded that either the mutagenic effect continued at very high doses but the increase in surviving revertants was too small to be detected, or the mutagenic effect did not continue at high doses. In either case, the final slope represents a killing effect only.

An interesting feature of the mutant yield curves obtained after saline incubation 
and after exposure to light is the rather extended plateau which connects the dose ranges which correspond to an increasing and decreasing yield. Again, alternative explanations would be either that the plateau is the result of an exact balance between the mutagenic and lethal effects as modified by post-treatment or that the plateau is due to a saturation of the mutagenic effect at low doses combined with a very broad shoulder on the survival curve for the organisms which become revertants.

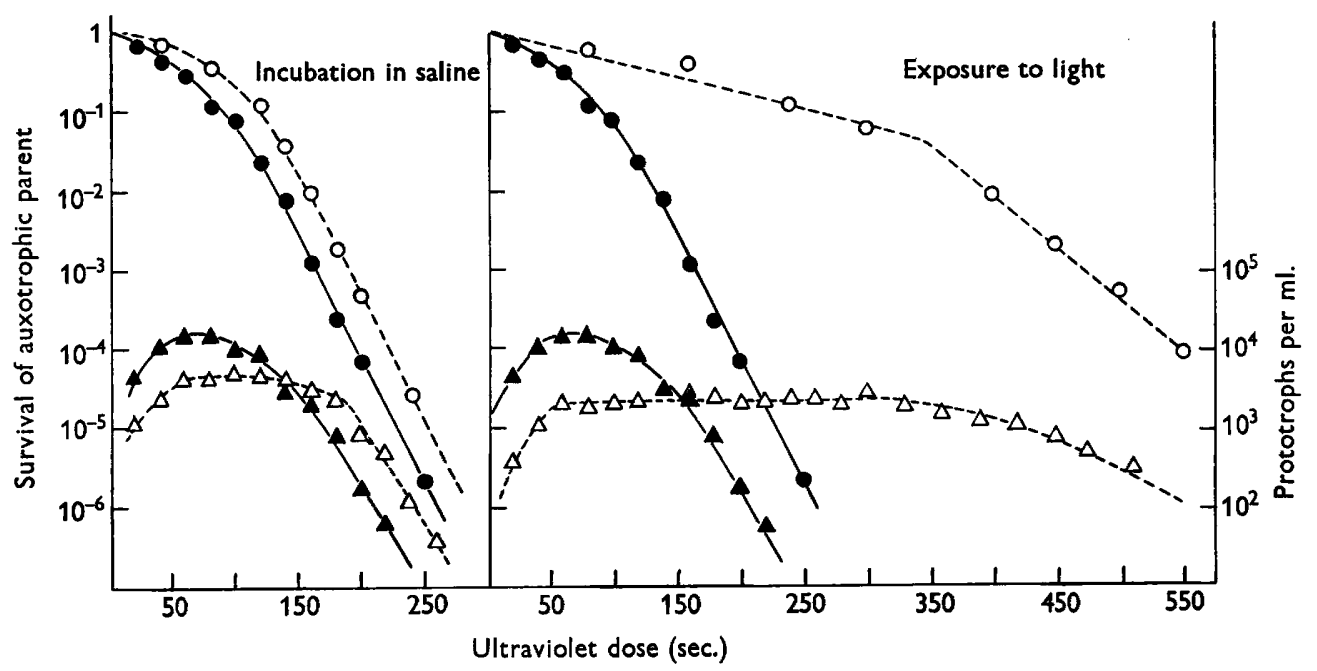

Fig. 1. Survival curves for the parent tryptophan-requiring strain Escherichia coli WP2 and absolute yields of prototrophs. Solid curves: no post-irradiation treatment; broken curves: post-irradiation incubation in saline and post-irradiation exposure to light. Circles = parent survival values; triangles = yields of prototrophs. All platings on SEM.

When irradiated organisms were plated on MM instead of SEM, only a small number of revertant colonies appeared. These originated from organisms which were capable of sufficient protein and RNA synthesis for fixation of the mutation and for phenotypic expression, without the necessity of supplying a pool of amino acids (Haas $\&$ Doudney, 1959). Figure 2 shows the dose-mutation curves for this small 'zeropoint' fraction of the total yield, as obtained by plating on MM immediately after irradiation and after $1 \mathrm{hr}$. of incubation in saline or $1 \mathrm{hr}$. of exposure to light. Plateaus appear in all of these cases. However, in contrast to the results for the major part of the yield, saline incubation appeared to increase the 'zero-point' yield after all doses of u.v.-radiation.

Further details concerning the latter effect are given in Table 1. In the case of Escherichia coli strain WP2, the increased yield obtained by saline incubation before plating was also obtained by simply plating a smaller number of bacteria. This dilution effect is usually ascribed to the existence of competitive suppression (Grigg, 1958). Since the plating was on MM, it is probable that the potential revertants competed with other surviving auxotrophic bacteria for nutrients released by non-survivors (Ryan, 1954). Presumably this release of nutrients also occurred during the incubation in liquid medium before plating. Therefore the 'zero-point' yield is probably not truly zero-point but derives from bacteria whose requirement 


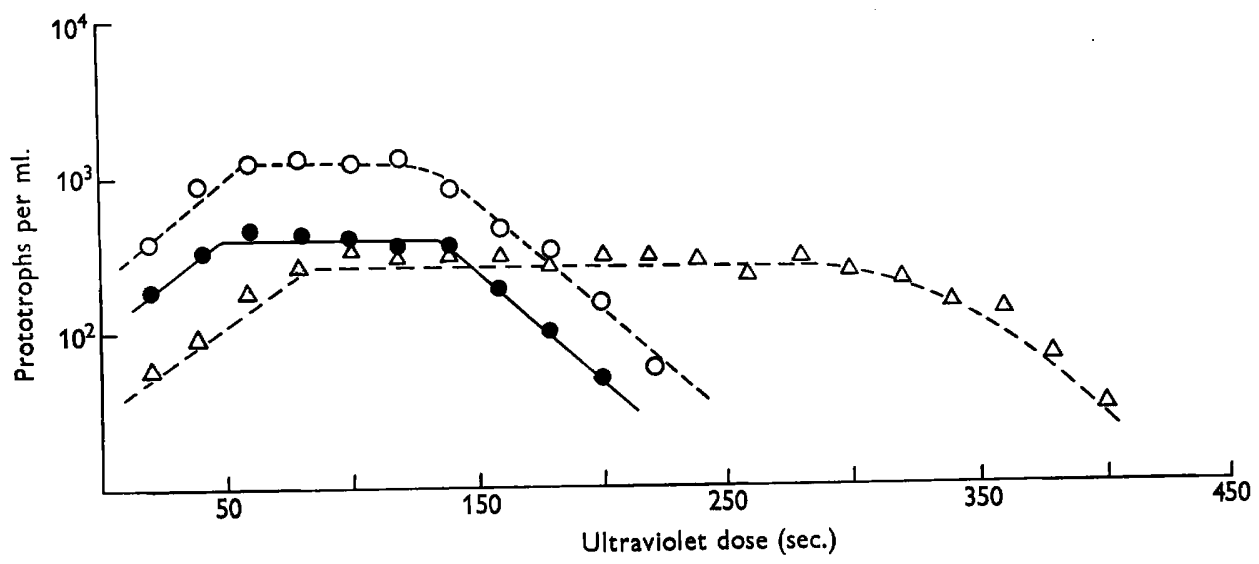

Fig. 2. Dose mutation curves obtained by plating on MM. Solid curve: no post-irradiation treatment; open circles $=$ post-irradiation incubation in saline ; open triangles $=$ post-irradiation exposure to light.

\section{Table 1. Effect of incubation in saline after u.v.-radiation on the zero-point yields of strains WP2 and WWP2}

The strains were grown in broth to a concentration of $2 \times 10^{9}$ per ml., centrifuged twice and re-suspended in saline. u.v. dose $=80$ sec. Volume plated $=0.1 \mathrm{ml}$. Plating on MM. Colonies counted after 8 days incubation at $37^{\circ}$.

Time of incubation in saline (hr.)

$\mathbf{0}$ 1
Dilution plated

$10^{-1}$

$10^{-2}$

$10^{-1}$

$10^{-2}$
No. of zero-point revertants per ml. of irradiated suspension

$\begin{array}{rr}\text { WP2 } & \text { WWP2 } \\ 1300 & 515 \\ 2550 & 800 \\ 2280 & 845 \\ 2800 & 1800\end{array}$

Table 2. Effect of prolonged aeration in broth before irradiation on revertant yield

An overnight broth culture of WP2 grown without aeration, was diluted to give $10^{3}$ cells per ml. in a total volume of $30 \mathrm{ml}$. This culture was grown by aeration. At various times, $8 \mathrm{ml}$. were removed, centrifuged and re-suspended in saline. One tenth of an ml. was diluted appropriately to determine the density of WP2 cells. The remaining $3 \mathrm{ml}$. were irradiated and plated. u.v. dose $=80$ sec. $\left(450\right.$ ergs per $\left.\mathrm{mm}^{2}\right)$.

\begin{tabular}{|c|c|c|c|c|}
\hline \multirow{2}{*}{$\begin{array}{l}\text { Time of } \\
\text { sampling } \\
\text { (hr.) }\end{array}$} & \multirow{2}{*}{$\begin{array}{c}\text { Density of } \\
\text { WP } 2 \text { cells } \\
\left(\times 10^{-9}\right)\end{array}$} & \multicolumn{2}{|c|}{$\begin{array}{c}\text { Revertants/ml. appearing after } \\
\text { u.v.-irradiation on }\end{array}$} & \multirow{2}{*}{$\% \frac{\mathbf{M M}}{\mathbf{S E M}}$} \\
\hline & & SEM & $\mathbf{M M}$ & \\
\hline 18 & 0.97 & 7,000 & 280 & $\mathbf{3} \cdot \boldsymbol{9}$ \\
\hline 20 & $1 \cdot 1$ & 10,800 & 320 & 3.0 \\
\hline 22 & $1 \cdot 3$ & 8,300 & 640 & $7 \cdot 6$ \\
\hline 24 & $2 \cdot 0$ & 9,150 & 960 & 10.5 \\
\hline 26 & $2 \cdot 2$ & 8,200 & 900 & $12 \cdot 5$ \\
\hline 28 & $2 \cdot 4$ & 9,000 & 1,280 & $14 \cdot 8$ \\
\hline 30 & $2 \cdot 4$ & 9,200 & 1,500 & $16 \cdot 3$ \\
\hline 43 & $2 \cdot 2$ & 3,500 & 1,100 & $29 \cdot 6$ \\
\hline
\end{tabular}


of an external supply of nutrients for fixation and phenotypic expression of the mutation was much smaller than the requirement of the rest of the population.

In the case of strain WWP2, the dilution effect was not completely eliminated by incubation in saline. This probably means that release of nutrients by non-survivors started during the $1 \mathrm{hr}$. incubation period in saline, and for this strain continued after plating.

A 'zero-point' yield of revertants is not obtained when the auxotrophic bacteria are irradiated while in the logarithmic phase of growth (Lieb, 1958). Table 2 shows that the 'zero-point' yield increased as the bacteria to be irradiated entered the late logarithmic phase. This yield reached a maximum at a later time than the rest of the yield and declined more slowly as the culture aged.

\section{DISCUSSION}

It has been suggested that the 'zero-point' yield may be due to storage of intracellular precursor material, thereby making the bacteria less dependent upon an external supply (Lieb, 1958). Since the total yield is not 'zero-point', it would seem that, if this suggestion is correct, the unirradiated stationary-phase population must be heterogeneous with respect to the size of the intracellular pool of precursors. This raises some interesting questions. If the yield obtained by plating directly on MM after irradiation comes from bacteria which have the largest pool, then the size of this small part of the yield is already determined before irradiation. This is in agreement with the second of the two possible interpretations of the dose/mutation curves mentioned previously, i.e. the mutagenic effect is saturated at low doses. A similar suggestion was proposed for the case of colour variants of Escherichia coli strain $B / r$ appearing after u.v.-irradiation (Newcombe \& Whitehead, 1951).

The major part of the yield appears only on supplemented MM, increasing with the amount of supplement to a maximum when the broth concentration is about 2.5-5\% (Witkin, 1956). By extending the case of the 'zero-point' yield to the remainder of the yield, it would appear that the number of revertants appearing on any given level of enrichment may also be pre-determined by the number of unirradiated cells which have a corresponding-sized pool of precursor material.

Why does the number of revertants not continue to increase when the broth concentration is increased to $100 \%$ ? The obvious answer is that the medium loses its selectivity since the surviving auxotrophic bacteria will undergo more and more divisions. In other words, the number of revertants that can be detected is limited by the method of detection.

Witkin showed that the critical period following irradiation, during which the yield can be influenced by treatments affecting protein (and RNA) synthesis, was proportional to the u.v. dose in the low dose-range. There is apparently a maximum dose in the low dose-range such that further irradiation no longer increases the sensitive period, presumably because there is no further delay in DNA synthesis (Witkin, 1958). Since $(a)$ it is the amount of protein synthesis which takes place before DNA synthesis which determines the observed yield, $(b)$ the relative amount of protein synthesis which takes place in a given bacterium is determined before irradiation, and $(c)$ the method of detection limits the amount of protein synthesis which can lead to an observable mutation, an apparent saturation of the mutagenic effect is readily understandable. 
These considerations also explain the appearance of plateaus in the dose/mutation curves. It was suggested above that if the mutagenic effect is saturated at a low dose of u.v. radiation, then the plateau might represent a broad shoulder on the survival curve of the bacteria which become revertants. The preceding analysis indicates that the fraction of potential prototrophs which is actually detected must arise in those auxotrophic bacteria which have a larger intracellular pool of precursors in comparison with those which are not detected. The size of this pool increases as the bacteria enter the stationary phase of the growth cycle. Gillies \& Alper (1960) found that the survival curves for Escherichia coli B/r (of which strain WP2 is a mutant) had the same final slopes when the bacteria were u.v.irradiated in the logarithmic and stationary phases. However, when the bacteria were in the logarithmic phase, the survival curve was a single exponential; when they were in the stationary phase, a shoulder appeared and the extrapolation number increased to about 3. Evidently, the more 'stationary phase' the bacteria are, the broader the shoulder on the survival curve. Thus the plateau on the dose/ mutation curve may simply be a reflection of the greater radiation-resistance of the most 'stationary phase' bacteria, i.e. the bacteria with the largest intracellular pools, the bacteria responsible for the observed yield.

Support for the above hypothesis might be obtained from investigation of the effect on the yield of mutations of experimental conditions, other than the use of bacteria in different phases of the growth cycle, which broaden the shoulder of the u.v. survival curve. Preliminary results obtained by growing cultures before irradiation in medium supplemented with a high concentration of glucose (Stapleton \& Engel, 1960) have already shown that the broadening of the shoulder of the survival curve is accompanied by a marked increase in the absolute yield of revertants.

The author wishes to express gratitude to $\mathrm{Dr}$ H. H. Rossi for frequent helpful discussions and Miss Barbara Potter for technical assistance. Based on work performed under Contract AT-(30-1)-2740 for the U.S. Atomic Energy Commission.

\section{REFERENCES}

DOUDNEy, C. O. (1961). Nucleic acid formation and ultraviolet light-induced mutation in bacteria: Some considerations in light of recent advances. J. cell. comp. Physiol. 58, suppl. 1, 145.

Doudney, C. O. \& HAas, F. L. (1958). Modification of ultraviolet-induced frequency and survival in bacteria by post-irradiation treatment. Proc. nat. Acad. Sci., Wash. 44, 390.

DoUdNEY, C. O. \& HAAS, F. L. (1960a). Some biochemical aspects of the postirradiation modification of ultra-violet-induced mutation frequency in bacteria. Genetics, 45, 1481.

Doudney, C. O. \& HaAs, F. L. (1960b). Chloramphenicol, nucleic acid synthesis and mutation induced by ultraviolet light. Biochim. biophys. Acta, 40, 375.

GILlIEs, N. E. \& ALPER, T. (1960). The nucleic acid content of Escherichia coli strains B and $\mathrm{B} / \mathrm{r}$. Biochim. biophys. Acta, 43, 182.

GRIGG, G. W. (1958). Competitive suppression and the detection of mutations in microbial populations. Aust. J. biol. Sci. 11, 69.

HAAS, F. L. \& Doudney, C. O. (1959). Mutation induction and expression in bacteria. Proc. nat. Acad. Sci., Wash. 45, 1620.

Hir., R. F. \& Srmson, E. (1961). A study of radiosensitive and radioresistant mutants of Escherichia coli strain B. J. gen. Microbiol. 24, 1.

LIEB, M. (1958). Relationships between mutation, phenotypic lag and protein synthesis. Proc. 10 int. Congr. Genetics, 2, 170. 
LIEB, M. (1960). Deoxyribonucleic acid synthesis and ultraviolet-induced mutation. Biochim. biophys. Acta, 37, 155.

Newcombe, H. B. \& Whitenead, H. A. (1951). Photoreversal of ultraviolet-induced mutagenic and lethal effects in Escherichia coli. J. Bact. 61, 243.

RYAN, F. J. (1954). The delayed appearance of mutants in bacterial cultures. Proc. nat. Acad. Sci., Wash. 40, 178.

Soboren, J. \& Nyc, J. F. (1961). Amino acid interactions in Neurospora crassa. J. Bact. $82,20$.

Stapleton, G. E. \& ENGEL, M. S. (1960). Cultural conditions as determinants of sensitivity of Escherichia coli to damaging agents. J. Bact. 80, 544.

Vogel, H. J. \& Bonner, D. M. (1956). Acetylornithase of Escherichia coli: Partial purification and some properties. J. biol. Chem. 218, 97.

WeATHERWAX, R. S. \& LANDMAN, O. E. (1960). Ultraviolet light-induced mutation and deoxyribonucleic acid synthesis in Escherichia coli. J. Bact. 80, 528.

WrTkın, E. M. (1956). Time, temperature, and protein synthesis: A study of ultravioletinduced mutation in bacteria. Cold Spring Harb. Symp. quant. Biol. 21, 123.

Witkin, E. M. (1958). Post-irradiation metabolism and the timing of ultraviolet-induced mutations in bacteria. Proc. 10th int. Congr. Genetics, 1, 280.

WrrkIN, E. M. (1961). Modification of mutagenesis initiated by ultraviolet light through posttreatment of bacteria with basic dyes. J. cell. comp. Physiol. 58, suppl. 1, 135. 